\title{
Patterns of dominant flows in the world trade web
}

\author{
M. Ángeles Serrano, ${ }^{1}$ Marián Boguñá, ${ }^{2}$ and Alessandro Vespignani ${ }^{3,4}$ \\ ${ }^{1}$ Institute of Theoretical Physics, LBS, FSB, EPFL, BSP 725 - Unil, 1015 Lausanne, Switzerland \\ ${ }^{2}$ Departament de Física Fonamental, Universitat de Barcelona, \\ Martí $i$ Franquès 1, 08028 Barcelona, Spain \\ ${ }^{3}$ School of Informatics, Indiana University, Eigenmann Hall, \\ 1900 East Tenth Street, Bloomington, IN 47406, USA \\ ${ }^{4}$ Complex Network Lagrange Laboratory (CNLL), \\ Institute for Scientific Interchange (ISI), Torino 10133, Italy
}

(Dated: today)

\begin{abstract}
The large-scale organization of the world economies is exhibiting increasingly levels of local heterogeneity and global interdependency. Understanding the relation between local and global features calls for analytical tools able to uncover the global emerging organization of the international trade network. Here we analyze the world network of bilateral trade imbalances and characterize its overall flux organization, unraveling local and global high-flux pathways that define the backbone of the trade system. We develop a general procedure capable to progressively filter out in a consistent and quantitative way the dominant trade channels. This procedure is completely general and can be applied to any weighted network to detect the underlying structure of transport flows. The trade fluxes properties of the world trade web determines a ranking of trade partnerships that highlights global interdependencies, providing information not accessible by simple local analysis. The present work provides new quantitative tools for a dynamical approach to the propagation of economic crises.
\end{abstract}

\section{INTRODUCTION}

The term "globalization", when applied to the international economic order, refers to the presence of an intricate network of economic partnership among an increasing number of countries [1. In this context, the International trade system, describing the fundamental exchange of goods and services, plays a central role as one of the most important interaction channels between states [2]. For instance, it broadly defines the substrate for the spreading of major economic crises [3, 4, 5], such as the 1997 Asiatic crisis [5, 6, 7, which shows how economic perturbations originated in a single country can somehow propagate globally in the world. Moreover, commercial trade flows are indeed highly correlated with other types of cross-country economic interactions (flows of services, financial assets, workers) and so stand as a good indicator for more general economic relations 8 . The International trade system as an independent extract of the world economy is therefore still a partial view of the whole system; a complete description would consider the feedback mechanisms that operate between international trade imbalances and other economic variables such as investment, debt, or currency prices. On the other hand, the study of the International trade network in a system's perspective represents a necessary first step before proceeding with a subsequent more integrative investigation and has proven to be successful in providing insight into some of its global properties.

The large size and the entangled connectivity pattern characterizing the international trade organization point out to a complex system whose properties depend on its global structure. In this perspective, it appears natural to analyze the world trade system at a global level, every country being important regardless of its size or wealth and fully considering all the trade relationships. A convenient framework for the analysis of complex interconnected systems is network analysis [9, 10. Within this approach, countries are represented as nodes and trade relationships among them as links. Such visualizations of bilateral trade relations have been used in recent years to help analyze gravity models [11, 12, often proposed to account for the world trade patterns and their evolution [13. While the first attempts to study the trade system as a complex network have successfully revealed a hierarchical organization 14, 15, 16, these studies focused on topological aspects neglecting fundamental components, such as the heterogeneity in the magnitude of the different bilateral trade relations and their asymmetry. These are essential issues in the understanding of the interplay between the underlying structure and the principles that rule the functional organization of the system.

Here we tackle the quantitative study of the world trade network by implementing the trade flux analysis at a global scale. To this end, we construct the weighted directed network of merchandize trade imbalances between world countries. In this representation, each country appears as a node and a directed link is drawn among any pair whenever a bilateral trade imbalance exists, i.e., whenever bilateral imports does not balance exports. The direction of the arrow follows that of the net flow of money and it is weighted according to the magnitude of the imbalance between the two countries. More precisely, we define the elements $E_{i j}$ that measure the exports of country $i$ to country $j$ and the elements $I_{i j}$ that measure the imports of country $i$ from country $j$. The trade imbalance matrix is therefore defined as $T_{i j}=E_{i j}-I_{i j}$ and 

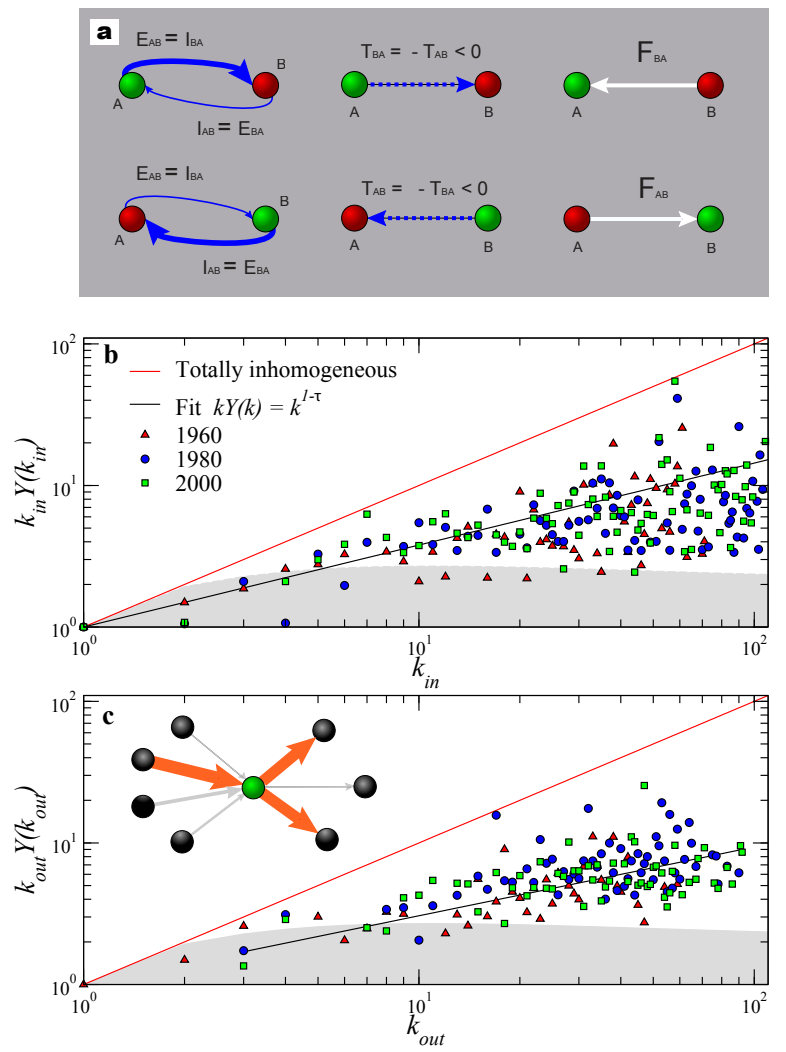

FIG. 1: Measuring local inhomogeneity in fluxes. a, conceptual representation of the link construction process. $\mathbf{b}$ and $\mathbf{c}$, local inhomogeneity for incoming (b) and outgoing (c) connections measured by $k Y(k)$ as compared to the null model. The diagonal line corresponds to the maximum possible inhomogeneity, with only one connection carrying all the flux. The line $k Y(k)=1$ is the maximum homogeneity, with all the fluxes equally distributed among the connections. The area depicted in grey corresponds to the average of $k Y(k)$ under the null model plus two standard deviations. The solid lines are the best fit estimates which give $k_{i n} Y\left(k_{i n}\right) \sim k_{i n}^{0.6}$ and $k_{\text {out }} Y\left(k_{\text {out }}\right) \sim k_{\text {out }}^{0.5}$. The inset in $(\mathbf{c})$ sketches a pathway through a country arising from strong local inhomogeneity in incoming and outgoing connections.

measures the net money flow from country $j$ to country $i$ due to trade exchanges. Since $E_{i j}=I_{j i}$ and $I_{i j}=E_{j i}$, $T$ is an antisymmetric matrix with $T_{i j}=-T_{j i}$, and a directed network can be easily constructed by assuming a directed edge pointing to the country with positive balance. The network of the net trade flows is therefore defined in terms of a weighted adjacency matrix $F$ with $F_{i j}=\left|T_{i j}\right|=\left|T_{j i}\right|$ for all $i, j$ with $T_{i j}<0$, and $F_{i j}=0$ for all $i, j$ with $T_{i j} \geq 0$ (see Fig. 1a for a pictorial description).

By using the above procedure we constructed the network of trade imbalances by using the data set which reports the annual merchandize trade activity between independent states in the world during the period 19482000 , together with the annual values of their Gross Do- mestic Product per capita and population figures (19502000) [17, 25]. The time span of the data set allows us to study the change of trade flow networks with yearly snapshots characterizing the time evolution of the trade system. The most basic topological characterization of each country within the network is given by the number of incoming and outgoing links, $k_{\text {in }}$ and $k_{\text {out }}$ respectively, which inform us about the number of neighboring countries that contribute positively and negatively to the net trade imbalance of the country under consideration. A precise assessment of the country trade balance cannot however neglect the magnitude of the fluxes carried by each trade relation. This information can be retrieved summing up all the weights of the incoming or outgoing links, which give us the total flux of money due to trade entering to or leaving from the country of interest. In the network literature, these two variables are called incoming and outgoing strength and are denoted by $s_{j}^{\text {in }}=\sum_{i} F_{i j}$ and $s_{j}^{\text {out }}=\sum_{i} F_{j i}$, respectively [18. The total trade imbalance of a country can then be computed as $\Delta s_{j}=s_{j}^{i n}-s_{j}^{\text {out }}$. Depending on $\Delta s_{j}$, countries can be then defined as net consumers and net producers. Net producers export more than they import, the total outcome being a trade surplus which corresponds to $\Delta s_{j}>0$, whereas net consumers export less than they import, the total outcome being a trade deficit which is indicated by $\Delta s_{j}<0$. Since one incoming link for a given country is always an outgoing link for another, the sum of all the countries' trade imbalances in the network must be zero. While the local balance is not conserved, we are therefore dealing with a closed system which is globally balanced (the total flux is conserved). Merchandizes, or equivalently money, flows in the system from country to country with the peculiarity that there is a global flow of money from consumer countries to producer ones.

\section{LOCAL HETEROGENEITY AND BACKBONE EXTRACTION}

The obtained networks show a high density of connections and heterogeneity of the respective fluxes among countries. Indeed, as the number of countries increases, so does the average number of trade partners, as well as the total flux of the system, which is seen to grow proportional to the aggregated world Gross Domestic Product [19. The overall flux organization at the global scale can be characterized by the study of the flux distribution. A first indicator of the system heterogeneity is provided by the probability distribution $P\left(F_{i j}\right)$ denoting the probability that any given link is carrying a flux $F_{i j}$. The observed distribution is heavy-tailed and spans approximately four orders of magnitude [19]. Such a feature implies that only a small percentage of all the connections in the network carry most of its total flow $F$ and that there is no characteristic flux in the system, with most of the fluxes below the average and some of them with a much higher value. This is however not totally 
TABLE I: Sizes of the backbones. Percentage of the original total weight $F$, number of nodes $N$ and links $E$ in the 1960 and 2000 imbalance networks that remain in the backbone as a function of the significance level $\alpha$.

\begin{tabular}{lcccccc}
\hline & \multicolumn{3}{c}{1960} & \multicolumn{3}{c}{2000} \\
\hline$\alpha$ & $\% \mathrm{~F}$ & $\% \mathrm{~N}$ & $\% \mathrm{E}$ & $\% \mathrm{~F}$ & $\% \mathrm{~N}$ & $\% \mathrm{E}$ \\
\hline 0.2 & 88 & 100 & 25 & 92 & 98 & 25 \\
0.1 & 83 & 100 & 19 & 87 & 98 & 19 \\
0.05 & 79 & 99 & 15 & 84 & 97 & 15 \\
0.01 & 69 & 92 & 9 & 75 & 96 & 10 \\
\hline
\end{tabular}

unexpected since a large scale heterogeneity is a typical feature of large-scale networks. In addition, the global heterogeneity could just be due to differences in the sizes of the countries, in their population and in their respective Gross Domestic Product. More interesting is therefore the characterization of the local heterogeneity; i.e. given all the connections associated to each given country, how is the flux distribution for each of them.

A local heterogeneity implies that only a few links carry the biggest proportion of the country's total in-flow or out-flow. Interestingly, such a heterogeneity would define specific pathways within the network that accumulate most of the total flux. In order to asses the effect of inhomogeneities at the local level, for each country $i$ with $k$ incoming or outgoing trade partners we calculate [20, 21]

$$
k Y_{i}(k)=k \sum_{j=1}^{k} p_{i j}^{2},
$$

where $k$ can be either $k_{\text {in }}$ or $k_{\text {out }}$ in order to discern between inhomogeneities in incoming and outgoing fluxes, and where the normalized fluxes of node $i$ with its neighbors are calculated as $p_{i j}=F_{j i} / s_{i}^{i n}$ for incoming connections and as $p_{i j}=F_{i j} / s_{i}^{\text {out }}$ for the outgoing ones. The function $Y_{i}(k)$ is extensively used in economics as a standard indicator of market concentration, referred as the Herfindahl-Hirschman Index or HHI 22, 23], and it was also introduced in the complex networks literature as the disparity measure 24]. In all cases, $Y_{i}(k)$ characterizes the level of local heterogeneity. If all fluxes emanating from or arriving to a certain country are of the same magnitude, $k Y_{i}(k)$ scales as 1 independently of $k$, whereas this quantity depends linearly on $k$ if the local flux is heterogeneously organized with a few main directions. Increasing deviations from the constant behavior are therefore indicating heterogeneous situations in which fluxes leaving or entering each country are progressively peaked on a small number of links with the remaining connections carrying just a small fraction of the total trade flow. On the other hand, the deviations from the constant behavior have to be expected for low values of $k$ and it is important to compare the obtained results with the deviations simply produced by statistical fluctuations. To this end, we introduce a null model for the distribution of flows among a given number of neighbors in order to assess, in a case by case basis, whether the observed inhomogeneity can just be due to fluctuations or it is really significant.

The null model with the maximum random homogeneity corresponds to the process of throwing $k-1$ points in a $[0,1]$ interval, so that the interval ends up divided in $\mathrm{k}$ sections of different lengths representing the different values assigned to the $k$ variables $p_{i j}$ in the random case. It can be analytically proved that the probability that one of these variables takes a particular value $x$ depends on $k$ and is

$$
\operatorname{Prob}\left\{x<p_{i j}<x+d x\right\}=(k-1)(1-x)^{k-2} d x .
$$

This probability density function can be used to calculate the statistics of $k Y_{N M}(k)$ for the null model. Both the average and the standard deviation are found to depend on $k$ :

$$
\begin{gathered}
\left\langle k Y_{N M}(k)\right\rangle=k\left\langle Y_{N M}(k)\right\rangle=\frac{2 k}{k+1} \\
\sigma^{2}\left(k Y_{N M}(k)\right)=k^{2}\left(\frac{20+4 k}{(k+1)(k+2)(k+3)}-\frac{4}{(k+1)^{2}}\right),
\end{gathered}
$$

so that each node in the network with a certain in or out degree should be compared to the corresponding null model depending on the appropriate $k$.

In Fig. 1, we show the empirical measures along with the region defined by the average value of the same quantity $k Y(k)$ plus two standard deviations as given by the null model (shadowed area in grey). For a homogeneously random assignment of weights, this quantity converges to a constant value for large $k$, which is clearly different from the observed empirical behavior. Most empirical values lie out of the null model domain, which proves that the observed heterogeneity is due to a well definite ordering principle and not to random fluctuations.

The direct fit of the data indicates that both in and out fluxes follow the scaling law $k Y_{i}(k) \propto k^{\beta}$ with $\beta_{\text {in }}=0.6$ for the incoming connections and $\beta_{\text {out }}=0.5$ for the outgoing ones (see Fig. 1). This scaling represents and intermediate behavior between the two extreme cases of perfect homogeneity or heterogeneity but clearly points out the existence of strong local inhomogeneities. The emerging picture is therefore consistent with the existence of major pathways of trade flux imbalances (thus money) that enters the country using its major incoming links and leaves it through its most inhomogeneous outgoing trade channels (see inset in Fig. 1c).

The analysis of the local inhomogeneities in the trade fluxes prompts to the presence of high-flux backbones, sparse subnetworks of connected trade fluxes carrying most of the total flux in the network. This backbone is necessarily encoding a wealth of information being the dominating structure of the trade system. It is also worth remarking that the local heterogeneity is not just encoded 

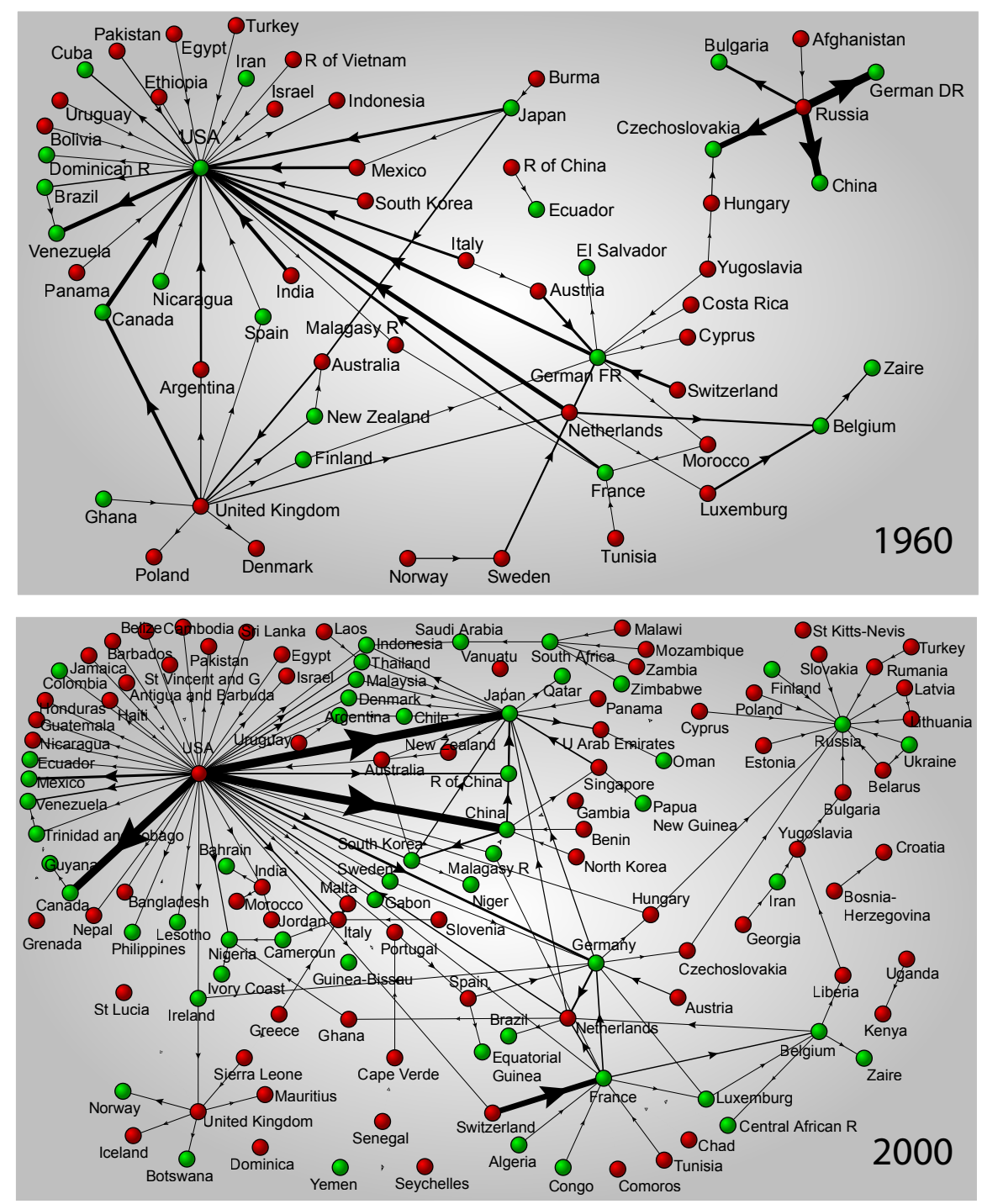

FIG. 2: Backbone of the world trade system. Snapshots of the $\alpha=10^{-3}$ backbone of the world trade imbalance web for the years 1960 and 2000. Notice that the most central economies are depicted at fixed positions to make both graphs more easily comparable.

in high flux links in terms of their absolute intensities, but also takes into account the local heterogeneity by comparing the strength of the fluxes associated to a given country with its total strength. It is then interesting to filter out this special links and provide snapshots of the trade system backbone. This can be achieved by comparing the link fluxes with the null model used for the calculation of the disparity in a pure random case. The same approach allows us the calculation for each connection of a country $i$ of the probability $\alpha_{i j}$ that its normalized flux value $p_{i j}$ is due to chance. Along these lines, we can identify highly inhomogeneous fluxes as those which satisfy

$$
\alpha_{i j}=1-(k-1) \int_{0}^{p_{i j}}(1-x)^{k-2} d x<\alpha
$$

where $\alpha$ is a fixed significance level. Notice that this ex- pression depends on the number of connections of each country, $k$. By choosing a global threshold for all countries we obtain a homogeneous criteria that allows us to compare inhomogeneities in countries with different number of connections and filter out links that carry fluxes which can be considered not compatible with a random distribution with an increasing statistical confidence. The backbone is then obtained by preserving all the links which beat the threshold for at least one of the two countries at the ends of the link while discounting the rest. By changing the significance level we can filter out the links focusing on progressively more relevant heterogeneities and backbones.

An important aspect of this new filtering algorithm is that it does not belittle small countries and then, it offers a systematic procedure to reduce the number of connec- 
tions without diminishing the number of countries and choosing the backbone according to the amount of trade flow we intend to characterize. It provides a quantitative and consistent way to progressively identify the relevant flow backbone once the level of statistical confidence with respect to the null case is fixed, or instead the total flow we want to represent in the system. Indeed, it is remarkable that when looking at the network of the year 2000 one finds that the $\alpha=0.05$ backbone contains only $15 \%$ of the original links yet accounting for $84 \%$ of the total trade imbalance. Most of the backbones form a giant connected component containing most of the countries in the network, and only for very high values of the confidence level, defining a sort of super-backbones, some disconnected components appear and the number of countries starts to drop. In this respect, the $\alpha=0.01$ backbone seems to offer the best trade-off since it keeps nearly all countries, $75 \%$ of the total trade imbalances, and one order of magnitude less connections than in the original network (see Table 1).

The backbone reduction is extremely effective in sorting out the most relevant part of the network and can be conveniently used for visualization purposes. For the sake of space and reproduction clarity, we report the backbones corresponding to $\alpha=10^{-3}$, still accounting for approximately $50 \%$ of the total flux of the system. Fig. 2 shows two snapshots of such backbones for 1960 and 2000. These high-flux backbones evidence geographical, political and historical relationships among countries which affect the observed trade patterns. For instance, the trade of US with its geographically closer neighbors and also continental neighbors, the case of Russia and the former Soviet republics, or France and its former colonies, the lack of strong trade relations between the two blocks in the cold war, more evident in 1960. In general terms, a recurrent motif present in all years is the star-like structure, formed by a central powerful economy surrounded by small dependent economies. The USA appears as one of those powerful hubs during all this period. However, other countries has gradually lost this role in favor of others. This is the case of the UK, which was the only star-like counterpart of the USA in 1948; since then its direct area of influence has been narrowing. On the contrary, other countries have arisen for different reasons as new hub economies. This is the case of some European countries, Japan, and most recently, China.

\section{DIFFUSION ON COMPLEX NETWORKS AND THE DOLLAR EXPERIMENT}

The picture emerging from our analysis has intriguing similarities with other directed flow networks, such as metabolic networks 21], that transport information, energy or matter. Indeed, the trade imbalances network can be seen as a directed flow network that transport money. In other words, we can imagine that net consumer countries are injecting money in the system. Money flows along the edges of the network to finally reach producer countries. Producer countries, however, do not absorb completely the incoming flux, redistributing part of it through the outgoing links. The network is therefore characterizing a complex dynamical process in which the net balance of incoming and outgoing money is the outcome of a global diffusion process. The realization of such a non-local dynamics in the flow of money due to the trade imbalances spurs the issue of what impact this feature might have on the effect that one economy can have on another. In order to tackle this issue we perform a simple numerical study, defined as the "dollar experiment". The "experiment" considers running on the networks two symmetric random walk processes. Since we are limited by the yearly frequency of the empirical data, we assume at first approximation that the time scale of the changes in the structure of the underlying trade imbalances network is bigger than the characteristic diffusion time of the random walk processes. In the first case we imagine that a consumer country $(\Delta s<0)$ is injecting one dollar from its net debit into the system. The dollar at this point travels through the network following fluxes chosen with a probability proportional to their intensity, and has as well a certain probability of being trapped in producer countries $(\Delta s>0)$ with a probability $P_{a b s}=\frac{\Delta s}{s_{i n}}$. More precisely, if we consider a consumer country, such as the USA, the traveling dollar goes from country to country always following outgoing fluxes chosen with a probability proportional to their intensity. If in its way it finds another source it just crosses it, whereas if it finds a producer country $j$ it has a probability $P_{a b s}(j)$ of being absorbed. Mathematically, this process is a random walk on a directed network with heterogeneous diffusion probability and in the presence of sinks. By repeating this process many times it is possible to obtain the probability $e_{i j}$ that the traveling dollar originated in the source $i$ is finally absorbed in the sink $j$. In other words, for each dollar that a source country $i$ adds to the system, $e_{i j}$ represents the fraction of that dollar that is retained in country $j$. The symmetric process considers that each producer country is receiving a dollar and the traveler dollar goes from country to country always following incoming links backward chosen with a probability proportional to their intensity. If in its way it finds another sink it just crosses it, whereas if it finds a source $j$ it has a probability $P_{a b s}(j)=\frac{|\Delta s|}{s_{\text {out }}}$ of remaining in that country. The iteration of this process gives the probability $g_{i j}$ that yields the fraction originated in the source country $j$ of each dollar that a sink country retains. Consequently, these two quantities are related by the detailed balance condition

$$
\left|\Delta s_{i}\right| e_{i j}=\Delta s_{j} g_{j i}
$$

The matrices $e_{i j}$ and $g_{j i}$ are normalized probability distributions and, therefore, they satisfy that $\sum_{j ; s i n k} e_{i j}=$ 1 and $\sum_{i ; \text { source }} g_{j i}=1$. Using this property in the de- 
TABLE II: Rankings from the Dollar experiment. Top: effect of two major source countries, USA and Switzerland, on the rest of the world. The first list is a top ten ranking of countries according to $e_{i j}$, where the index $i$ stands for the analyzed source. The second list is the top ten ranking of direct bilateral trade measured as the percentage of flux from the source country, that is, $e_{i j}^{\text {local }}=F_{i j} / s_{i}^{\text {out }}$. Bottom: major contributors to two major sink countries, Japan and Russia. The first list is a top ten ranking of countries according to $g_{i j}, i$ standing for the analyzed sink. The second list is the top ten ranking due to direct trade. In this case, the direct contribution is $g_{i j}^{l o c a l}=F_{j i} / s_{i}^{i n}$. Countries in boldface have no direct connection with the analyzed country. The values for $e_{i j}$ and $g_{i j}$ are obtained from the simulation of the dollar experiment described in the text using $10^{6}$ different realizations for each country, for the year 2000.

\begin{tabular}{|c|c|c|c|c|c|c|c|}
\hline \multicolumn{8}{|c|}{ Net Consumers - Sources } \\
\hline \multicolumn{4}{|c|}{ USA } & \multicolumn{4}{|c|}{ Switzerland } \\
\hline \multicolumn{2}{|c|}{ Dollar experiment } & \multicolumn{2}{|c|}{ Bilateral trade } & \multicolumn{2}{|c|}{ Dollar experiment } & \multicolumn{2}{|c|}{ Bilateral trade } \\
\hline Japan & $19.5 \%$ & Japan & $17.2 \%$ & France & $27.3 \%$ & France & $75.0 \%$ \\
\hline Canada & $9.9 \%$ & China & $16.7 \%$ & Germany & $10.0 \%$ & Germany & $9.5 \%$ \\
\hline China & $9.3 \%$ & Canada & $15.6 \%$ & Russia & $9.7 \%$ & Russia & $4.1 \%$ \\
\hline Saudi Arabia & $6.1 \%$ & Mexico & $5.1 \%$ & Japan & $8.5 \%$ & Netherlands & $2.6 \%$ \\
\hline Russia & $5.4 \%$ & Germany & $4.8 \%$ & Ireland & $6.9 \%$ & Ireland & $2.3 \%$ \\
\hline Germany & $4.5 \%$ & $\mathrm{R}$ of China & $3.1 \%$ & Norway & $6.0 \%$ & Belgium & $1.7 \%$ \\
\hline Indonesia & $4.3 \%$ & Italy & $3.0 \%$ & Saudi Arabia & $4.2 \%$ & Italy & $1.2 \%$ \\
\hline Malaysia & $3.9 \%$ & Venezuela & $2.8 \%$ & China & $3.4 \%$ & Austria & $1.1 \%$ \\
\hline Ireland & $2.7 \%$ & South Korea & $2.4 \%$ & Indonesia & $2.3 \%$ & Libya & $0.4 \%$ \\
\hline South Korea & $2.7 \%$ & Malaysia & $2.4 \%$ & Malaysia & $1.9 \%$ & Nigeria & $0.4 \%$ \\
\hline \multicolumn{8}{|c|}{ Net Producers - Sinks } \\
\hline \multicolumn{4}{|c|}{ Japan } & \multicolumn{4}{|c|}{ Russia } \\
\hline \multicolumn{2}{|c|}{ Dollar experiment } & \multicolumn{2}{|c|}{ Bilateral trade } & \multicolumn{2}{|c|}{ Dollar experiment } & \multicolumn{2}{|c|}{ Bilateral trade } \\
\hline USA & $62.6 \%$ & USA & $40.2 \%$ & USA & $33.3 \%$ & Germany & $9.0 \%$ \\
\hline UK & $7.3 \%$ & $\mathrm{R}$ of China & $9.3 \%$ & UK & $7.2 \%$ & Italy & $8.1 \%$ \\
\hline Spain & $3.8 \%$ & Singapore & $7.0 \%$ & Switzerland & $7.1 \%$ & USA & $7.7 \%$ \\
\hline Switzerland & $3.3 \%$ & South Korea & $5.6 \%$ & Poland & $7.0 \%$ & China & $5.9 \%$ \\
\hline Singapore & $2.4 \%$ & Germany & $5.1 \%$ & Turkey & $6.9 \%$ & Poland & $5.4 \%$ \\
\hline Turkey & $2.1 \%$ & UK & $4.8 \%$ & Spain & $5.1 \%$ & Japan & $4.4 \%$ \\
\hline Panama & $2.1 \%$ & Netherlands & $4.8 \%$ & Greece & $3.5 \%$ & Turkey & $4.3 \%$ \\
\hline Greece & $1.9 \%$ & China & $3.9 \%$ & Egypt & $2.2 \%$ & Switzerland & $4.0 \%$ \\
\hline Portugal & $1.5 \%$ & Mexico & $2.1 \%$ & Lithuania & $2.0 \%$ & Netherlands & $4.0 \%$ \\
\hline Egypt & $1.5 \%$ & Thailand & $2.1 \%$ & Portugal & $1.9 \%$ & UK & $3.6 \%$ \\
\hline
\end{tabular}

tailed balance condition, we can write

$$
\Delta s_{j}=\sum_{i: s o u r c e} e_{i j}\left|\Delta s_{i}\right| \quad \text { and } \quad\left|\Delta s_{i}\right|=\sum_{j: s i n k} g_{j i} \Delta s_{j}
$$

Then, the total trade imbalance of a sink or source country can be written as a linear combination of the trade imbalances of the rest of the source or sink countries, respectively. Therefore, by measuring $e_{i j}$, it is possible to discriminate the effect that one economy has on another or, with $g_{i j}$, to find out which consumer country is contributing the most to a producer one, in both cases taking into account the whole topology of the network and the inhomogeneities of the fluxes. The advantage of this approach lies on its simplicity and the lack of tunable parameters. Indeed, all the information is contained in the network itself, without assuming any kind of mod- eling on the influences among countries.

By using this experiment it is possible to evaluate for a consumer country where the money spent is finally going. For each dollar spent we know which percentage is going to any other producer country and we can rank those accordingly. It is important to remark that in this case countries might not be directly connected since the money flows along all possible paths, sometimes through intermediate countries. This kind of ranking is therefore different from the customarily considered list of the first neighbors ranked by magnitude of fluxes. The analysis indeed shows unexpected results and, as it has been already pointed out in other works [5] applying other methodologies, a country can have a large impact on other countries despite being a minor or undirect trading partner, see Table 2. Similarly, producer countries may have a share of the expenditure of non directly con- 
nected countries resulting in a very different ranking of their creditors. As an example, for each net dollar that the USA inject into the system, only $9.3 \%$ is retained in China although the direct connection imbalance between these countries is $16.7 \%$. Very interestingly, we find that Switzerland spend a large share of his trade imbalance in countries which do not have appreciable trade with it and are therefore not directly connected such as Japan, Indonesia, and Malaysia. The Swiss dollars go to these countries after a long path of trade exchanges mediated by other countries. By focusing on producer countries we find other striking evidence. While the first importer from Russia by looking locally at the ranking of trade imbalances is Germany, the global analysis shows that one third of all the money Russia gains from trade is coming directly or undirectly from the USA. In Table 2, we report other interesting anomalies detected by the global analysis.

\section{CONCLUSIONS}

In summary, we have introduced a novel quantitative approach applicable to any dense weighted complex network which filters out the dominant backbones while preserving most of the nodes in the original connected com- ponent. We have also discussed the behavior of a coupled dynamical process, the dollar experiment, which unveils the global properties of economic and trade partnerships. In a globalized economy, we face ever increasing problems in disentangling the complex set of relations and causality that might lead to crisis or increased stability. Focusing on just the bilateral relations among country economies is a reductionist approach that cannot work in a highly interconnected complex systems. We have proposed the use of the trade network representation and mathematical tools that allow to uncover some basic ordering emerging from the global behavior and the inclusion of nonlocal effects in the analysis of trade interdependencies. Future work on this grounds might help in the assessment of world trade relations and the understanding of the global dynamics underlying major economic crises.

\section{Acknowledgments}

We thank F. Vega-Redondo for useful comments. M. B. acknowledges financial support by DGES grant No. FIS2004-05923-CO2-02 and Generalitat de Catalunya grant No. SGR00889. A.V. is partially supported by the NSF award IIS-0513650.
[1] M. A. Centeno, A. Cooke, and S. R. Curran, NetMap Combined Studies, Mapping Globalization Project (Princeton University and University of Washington, 2006).

[2] P. R. Krugman, Brookings Papers on Economic Activity 1995, 327 (1995).

[3] R. Glick and A. Rose, J. of Intl. Money and Finance 18, 603 (1999).

[4] K. Forbes, in In Sebastian Edwards and Jeffrey Frankel (eds.), Preventing Currency Crises in Emerging Markets (University of Chicago Press, Chicago, 2002), pp. 77-124.

[5] T. Abeysinghe and K. Forbes, Rev. of Intl. Econ. 13, 356 (2005).

[6] M. Goldstein, The Asian Financial Crisis: Causes, Cures and Systemic Implications (International Institute for Economics, Washington, D. C., 1998).

[7] M. Noland, L.-G. Liu, S. Robinson, and Z. Wang, Global Economics Effects of the Asian Currency Devaluations (International Institute for Economics, Washington, D. C., 1998).

[8] P. R. Krugman and M. Obstfeld, International Economics: Theory and Policy, Seventh Edition (AddisonWesley, Lebanon, Indiana, U.S.A., 2005).

[9] R. Albert and A.-L. Barabási, Reviews of Modern Physics 74, 47 74, 47 (2002).

[10] S. N. Dorogovtsev and J. F. F. Mendes, Evolution of networks: From biological nets to the Internet and $W W W$ (Oxford University Press, Oxford, 2003).

[11] L. Krempel and T. Plümper, J. of World-Systems Research 5, 487 (1999).

[12] L. Krempel and T. Plümper, J. of Social Structures 4, 1
(2003).

[13] J. H. Bergstrand, The Review of Economics and Statistics 67, 474 (1985).

[14] M. A. Serrano and M. Bogunáa, Phys. Rev. E 68, 015101(R) (2003).

[15] D. Garlaschelli and M. I. Loffredo, Phys. Rev. Lett. 93, 188701 (2004).

[16] D. Garlaschelli and M. I. Loffredo, Physica A 355, 138144 (2005).

[17] K. S. Gleditsch, J. Conflict Resolut. 46, 712724 (2002).

[18] A. Barrat, M. Barthélemy, R. Pastor-Satorras, and A. Vespignani, Proc. Natl. Acad. Sci. USA 101, 3747 (2004).

[19] M. A. Serrano, J. Stat. Mech. p. L01002 (2007).

[20] M. Barthélemy, B. Gondran, and E. Guichard, Physica A 319, 633 (2003).

[21] E. Almaas, B. Kovács, T. Vicsek, Z. N. Oltvai, and A.-L. Barabási, Nature 427, 839 (2004).

[22] O. C. Herfindahl, Copper Costs and Prices: 1870-1957 (John Hopkins University Press, Baltimore, MD, USA, 1959).

[23] A. O. Hirschman, American Economic Review 54, 761 (1964).

[24] B. Derrida and H. Flyvbjerg, J. Phys. A 20, 5273 (1987).

[25] (version (4.1),

http://weber.ucsd.edu/ kgledits/exptradegdp.html)

The following issues should be considered: i) This expanded trade database includes additional estimates for missing values. ii) The definition of state in the international system is as defined by the Correlates of War Project (http://www.correlatesofwar.org/). iii) The 
figures for trade flows are in millions of current-year US dollars. iv) The import/export values correspond to exchanges of merchandizes. 